\title{
Izergin-Korepin Analysis on the Projected Wavefunctions of the Generalized Free-Fermion Model
}

\author{
Kohei Motegi \\ Faculty of Marine Technology, Tokyo University of Marine Science and Technology, Etchujima 2-1-6, Koto-Ku, Tokyo 135-8533, Japan \\ Correspondence should be addressed to Kohei Motegi; motegi@gokutan.c.u-tokyo.ac.jp
}

Received 17 April 2017; Accepted 21 May 2017; Published 20 June 2017

Academic Editor: Andrei D. Mironov

Copyright (c) 2017 Kohei Motegi. This is an open access article distributed under the Creative Commons Attribution License, which permits unrestricted use, distribution, and reproduction in any medium, provided the original work is properly cited.

We apply the Izergin-Korepin analysis to the study of the projected wavefunctions of the generalized free-fermion model. We introduce a generalization of the $L$-operator of the six-vertex model by Bump-Brubaker-Friedberg and Bump-McNamara-Nakasuji. We make the Izergin-Korepin analysis to characterize the projected wavefunctions and show that they can be expressed as a product of factors and certain symmetric functions which generalizes the factorial Schur functions. This result can be seen as a generalization of the Tokuyama formula for the factorial Schur functions.

\section{Introduction}

Integrable lattice models [1-4] are special classes of models in statistical physics in which many exact calculations are believed to be able to be done. The most local object in integrable models is called the $R$-matrix, and its mathematical structure was revealed in the mid-1980s [5, 6]. The underlying mathematical structure was named as the quantum groups, and the investigation of the quantum groups naturally leads to immediate constructions of various $R$-matrices.

From the point of view of statistical physics, $R$-matrices are the most local objects, and the study on the $R$-matrices is a starting point. The most important objects in statistical physics are partition functions. For the case of integrable models, partition functions are objects constructed from multiple $R$-matrices and are determined by boundary conditions. One of the most famous partition functions in integrable lattice models are the domain wall boundary partition functions which was first introduced and analyzed in $[7,8]$. In recent years, a more general class of partition functions which we shall call as the projected wavefunctions are attracting attention in its relation with algebraic combinatorics. The projected wavefunctions are the projection of the off-shell Bethe vector of integrable models into a class of some simple states labelled by the sequences of the particles or down spins. For the case of the free-fermion model in an external field, it was first shown by Bump-Brubaker-Friedberg [9] that the projected wavefunctions give a natural realization of the Tokuyama combinatorial formula for the Schur functions [10], which is a one-parameter deformation of the Weyl character formula (note that there are pioneering works using the free-fermion model implicitly in [11-13], and the Izergin-Korepin analysis and observation of the factorization phenomena on the domain wall boundary partition functions of the related models are called the Perk-Schultz (supersymmetric vertex) model [14] and the Felderhof freefermion model [15] in [16, 17]. There is also an application to the correlation functions in [18]). This observation triggered studies on finding various generalizations and variations of the Tokuyama-type formula for symmetric functions [19-27] such as the factorial Schur functions and symplectic Schur functions, and an interesting notion was introduced furthermore which the number theorists call it the metaplectic ice.

In this paper, we analyze the free-fermion model using the method initiated by Izergin-Korepin $[7,8]$. The method was developed by them in order to find the explicit expression of polynomials representing the domain wall boundary partition functions of the $U_{q}\left(s l_{2}\right)$ six-vertex model, from which the famous Izergin-Korepin determinant formula was found. The Izergin-Korepin analysis is the important method to study variants of the domain wall boundary partition functions. For example, it was applied to the domain wall boundary partition functions of the $U_{q}\left(s l_{2}\right)$ six-vertex model with reflecting 
end by Tsuchiya [28] to find its determinant formula. Extending the Izergin-Korepin analysis to more general class of partition functions is also important. Wheeler [29] invented a method to extend the Izergin-Korepin analysis on a class of partition functions called the scalar products. And in our very recent work [30], we extended the Izergin-Korepin analysis to study the projected wavefunctions of the $U_{q}\left(s l_{2}\right)$ six-vertex model. The resulting symmetric polynomials representing the projected wavefunctions contains the Grothendieck polynomials as a special case when the six-vertex model reduces to the five-vertex model [31-33]. We apply this technique to study the free-fermion model in an external field. To this end, we first introduce an ultimate generalization of the $L$ operator by introducing the inhomogeneous parameters and factorial parameters. We use an inhomogeneous version of the generalized $L$-operator in our forthcoming paper [34] having two types of factorial parameters, which generalizes the factorial $L$-operator by Bump-McNamara-Nakasuji [22]. We next view the projected wavefunctions as a function of the inhomogeneous parameters and characterize its properties by using the Izergin-Korepin analysis. We then show that the product of factors and certain symmetric functions satisfies all the required properties the projected wavefunctions must satisfy. The result is a generalization of $[9,22]$ and hence can be viewed as a generalization of the Tokuyama for the factorial Schur functions. The Izergin-Korepin analysis views the partition functions as functions of inhomogeneous parameters in the quantum spaces, whereas the arguments initiated in [9] view the partition functions as functions of the free parameter in the auxiliary spaces. The comparison of the two different ways of arguments seems to be interesting.

We will use the results of the projected wavefunctions to the algebraic combinatorial study of the generalized Schur functions [34]. For example, two ways of evaluations of the same partition functions can lead to integrable model constructions of algebraic identities of the symmetric functions. For example, two ways of evaluations of the domain wall boundary partition functions, a direct evaluation and an indirect evaluation using the completeness relation and the projected wavefunctions, can give rise to the dual Cauchy formula of the generalized Schur functions. This idea can also be applied to partition functions of integrable models under reflecting boundary to give dual Cauchy identities of the generalized symplectic Schur functions. Further detailed
Izergin-Korepin analysis on the domain wall boundary partition functions and the dual projected wavefunctions are required for the studies.

There are also studies on deriving Cauchy identities using the domain wall boundary partition functions like an intertwiner, invented in [35]. Deriving algebraic combinatorial properties of symmetric functions using their integrable model realizations is an active line of research. See [36-40] for more examples on Cauchy-type identities and more recent studies on the Littlewood-Richardson coefficients by [33, 41].

In any case, in order to conduct these studies, we first of all have to find out what are the explicit functions representing the projected wavefunctions. We think the Izergin-Korepin analysis presented in this paper is a fairly simple way to find out the explicit forms.

This paper is organized as follows. In the next section, we first list the generalized $L$-operator and introduce the projected wavefunctions. In Section 3, we make the IzerginKorepin analysis and list the properties needed to determine the explicit form of the projected wavefunctions. In Section 4, we show that the product of factors and certain symmetric functions satisfies all the required properties extracted from the Izergin-Korepin analysis, which means that the product is the explicit form of the projected wavefunctions. Section 5 is devoted to the conclusion of this paper.

\section{The Generalized Free-Fermion Model and the Projected Wavefunctions}

The most fundamental objects in integrable lattice models are the $R$-matrices and $L$-operators. The $R$-matrix of the freefermion model we treat in this paper is given by

$$
R_{a b}(z)=\left(\begin{array}{cccc}
1+t z & 0 & 0 & 0 \\
0 & t(1-z) & t+1 & 0 \\
0 & (t+1) z & z-1 & 0 \\
0 & 0 & 0 & z+t
\end{array}\right)
$$

acting on the tensor product $W_{a} \otimes W_{b}$ of the complex twodimensional space $W_{a}$.

The $L$-operator of the free-fermion model we use as bulk pieces of the projected wavefunctions in this paper is given by

$$
L_{a j}\left(z, w_{j}, \alpha_{j}, \gamma_{j}\right)=\left(\begin{array}{cccc}
w_{j}-\gamma_{j} z & 0 & 0 & 0 \\
0 & t w_{j}+\gamma_{j} z & w_{j} & 0 \\
0 & (t+1) z & \alpha_{j} w_{j}+\left(1-\alpha_{j} \gamma_{j}\right) z & 0 \\
0 & 0 & 0 & -t \alpha_{j} w_{j}+\left(1-\alpha_{j} \gamma_{j}\right) z
\end{array}\right) \text {, }
$$

acting on the tensor product $W_{a} \otimes \mathscr{F}_{j}$ of the space $W_{a}$ and the two-dimensional Fock space at the $j$ th site $\mathscr{F}_{j}$.
The parameters $w_{j}, \alpha_{j}$, and $\gamma_{j}$ can be regarded as parameters associated with the quantum space $\mathscr{F}_{j}$. The $L$-operators 


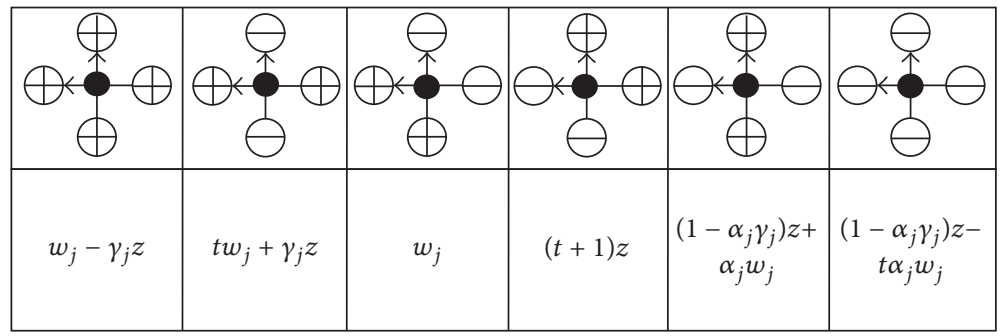

FIgURE 1: The $L$-operator $L_{a j}\left(z, w_{j}, \alpha_{j}, \gamma_{j}\right)(2)$. The horizontal line is the space $W_{a}$, and the vertical line is the space $\mathscr{F}_{j}$.

giving the Schur functions [9] and factorial Schur functions [22] are a special limit of the generalized $L$-operator (2) given by

$$
\begin{aligned}
L_{a j}(z, 1,0,0) & =\left(\begin{array}{cccc}
1 & 0 & 0 & 0 \\
0 & t & 1 & 0 \\
0 & (t+1) z & z & 0 \\
0 & 0 & 0 & z
\end{array}\right), \\
L_{a j}\left(z, 1, \alpha_{j}, 0\right) & =\left(\begin{array}{cccc}
1 & 0 & 0 & 0 \\
0 & t & 1 & 0 \\
0 & (t+1) z & \alpha_{j}+z & 0 \\
0 & 0 & 0 & -t \alpha_{j}+z
\end{array}\right),
\end{aligned}
$$

respectively.

The $L$-operator (2) together with the $R$-matrix (1) satisfies the $R L L$ relation:

$$
\begin{aligned}
& R_{a b}\left(\frac{z_{1}}{z_{2}}\right) L_{a j}\left(z_{1}, w_{j}, \alpha_{j}, \gamma_{j}\right) L_{b j}\left(z_{2}, w_{j}, \alpha_{j}, \gamma_{j}\right) \\
& \quad=L_{b j}\left(z_{2}, w_{j}, \alpha_{j}, \gamma_{j}\right) L_{a j}\left(z_{1}, w_{j}, \alpha_{j}, \gamma_{j}\right) R_{a b}\left(\frac{z_{1}}{z_{2}}\right),
\end{aligned}
$$

acting on $W_{a} \otimes W_{b} \otimes \mathscr{F}_{j}$.

Let us denote the orthonormal basis of $W_{a}$ and its dual as $\left\{|0\rangle_{a},|1\rangle_{a}\right\}$ and $\left\{{ }_{a}\langle 0|,{ }_{a}\langle 1|\right\}$ and the orthonormal basis of $\mathscr{F}_{j}$ and its dual as $\left\{|0\rangle_{j},|1\rangle_{j}\right\}$ and $\left\{{ }_{j}\langle 0|,{ }_{j}\langle 1|\right\}$. The matrix elements of the $L$-operator can be written as ${ }_{a}\left\langle\left.\gamma\right|_{j}\left\langle\delta\left|L_{a j}\left(z, w_{j}, \alpha_{j}, \gamma_{j}\right)\right| \alpha\right\rangle_{a} \mid \beta\right\rangle_{j}$, which we will use this form in the next section. See Figure 1 for a pictorial description of the $L$-operator (2).

The $R$-matrices and the $L$-operators have origins in statistical physics, and $|0\rangle$ or its dual $\langle 0|$ can be regarded as a hole state, while $|1\rangle$ or its dual $\langle 1|$ can be interpreted as a particle state from the point of view of statistical physics. We sometimes use the terms hole states and particle states to describe states constructed from $|0\rangle,\langle 0|| 1$,$\rangle , and \langle 1|$, since they are convenient for the description of the states. In the quantum inverse scattering method, the Fock spaces $W_{a}$ and $\mathscr{F}_{j}$ are usually called the auxiliary and quantum spaces, respectively.
For later convenience, we also define the following Pauli spin operators $\sigma^{+}$and $\sigma^{-}$as operators acting on the (dual) orthonormal basis as

$$
\begin{aligned}
& \sigma^{+}|1\rangle=|0\rangle, \\
& \sigma^{+}|0\rangle=0, \\
& \langle 0| \sigma^{+}=\langle 1|, \\
& \langle 1| \sigma^{+}=0, \\
& \sigma^{-}|0\rangle=|1\rangle, \\
& \sigma^{-}|1\rangle=0, \\
& \langle 1| \sigma^{-}=\langle 0|, \\
& \langle 0| \sigma^{-}=0 .
\end{aligned}
$$

To construct projected wavefunctions, we introduce the monodromy matrix $T_{a}\left(z \mid w_{1}, \ldots, w_{M}\right)$ (Figure 2(a)) from the generalized $L$-operator (2) as

$$
\begin{aligned}
T_{a} & \left(z \mid w_{1}, \ldots, w_{M}\right) \\
& =L_{a M}\left(z, w_{M}, \alpha_{M}, \gamma_{M}\right) \cdots L_{a 1}\left(z, w_{1}, \alpha_{1}, \gamma_{1}\right) \\
& =\left(\begin{array}{ll}
A\left(z \mid w_{1}, \ldots, w_{M}\right) & B\left(z \mid w_{1}, \ldots, w_{M}\right) \\
C\left(z \mid w_{1}, \ldots, w_{M}\right) & D\left(z \mid w_{1}, \ldots, w_{M}\right)
\end{array}\right)_{a} \\
& \in \operatorname{End}\left(W_{a} \otimes \mathscr{F}_{1} \otimes \cdots \otimes \mathscr{F}_{M}\right) .
\end{aligned}
$$

The matrix elements $A\left(z \mid w_{1}, \ldots, w_{M}\right), B\left(z \mid w_{1}, \ldots\right.$, $\left.w_{M}\right), C\left(z \mid w_{1}, \ldots, w_{M}\right)$, and $D\left(z \mid w_{1}, \ldots, w_{M}\right)$ are called the $A B C D$ operators, which are $2^{M} \times 2^{M}$ matrices acting on the tensor product of the quantum spaces $\mathscr{F}_{1} \otimes \cdots \otimes \mathscr{F}_{M}$.

To create projected wavefunctions, what is important is the $B$-operator $B\left(z \mid w_{1}, \ldots, w_{M}\right)$ (Figure $\left.2(\mathrm{~b})\right)$ which has the role of creating particles in the quantum spaces $\mathscr{F}_{1} \otimes \cdots \otimes \mathscr{F}_{M}$. We next introduce the following state vector.

$\left|\Phi_{M, N}\left(z_{1}, \ldots, z_{N} \mid w_{1}, \ldots, w_{M}\right)\right\rangle \in \mathscr{F}_{1} \otimes \cdots \otimes \mathscr{F}_{M}$ uses the $B$-operators as

$$
\begin{aligned}
& \left|\Phi_{M, N}\left(z_{1}, \ldots, z_{N} \mid w_{1}, \ldots, w_{M}\right)\right\rangle \\
& \quad=B\left(z_{1} \mid w_{1}, \ldots, w_{M}\right) \cdots B\left(z_{N} \mid w_{1}, \ldots, w_{M}\right)|\Omega\rangle_{M},
\end{aligned}
$$

where $|\Omega\rangle_{M}:=|0\rangle_{1} \otimes \cdots \otimes|0\rangle_{M} \in \mathscr{F}_{1} \otimes \cdots \otimes \mathscr{F}_{M}$ is the vacuum state in the tensor product of quantum spaces. 


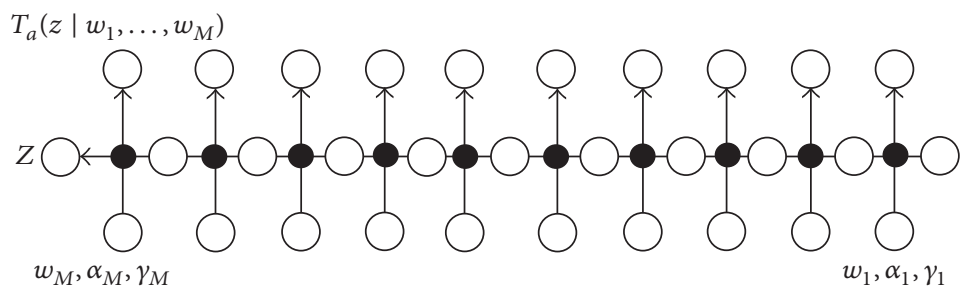

(a)

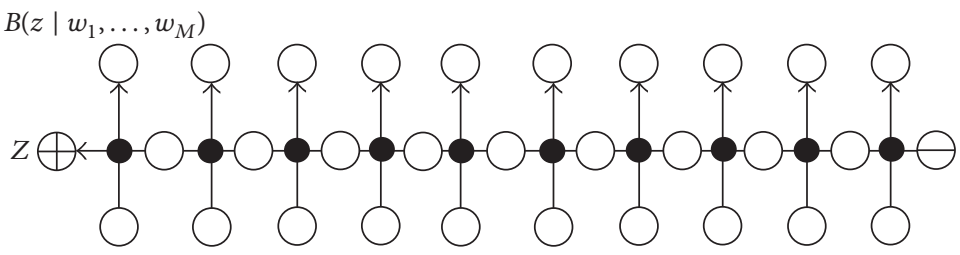

(b)

Figure 2: The monodromy matrix $T_{a}\left(z \mid w_{1}, \ldots, w_{M}\right)$ (7) (a) and the $B$-operator $B\left(z \mid w_{1}, \ldots, w_{M}\right)$ (b).

Due to the so-called ice rule of the $L$-operator ${ }_{a}\left\langle\left.\gamma\right|_{j}\left\langle\delta\left|L_{a j}\left(z, w_{j}, \alpha_{j}, \gamma_{j}\right)\right| \alpha\right\rangle_{a} \mid \beta\right\rangle_{j}=0$ unless $\alpha+\beta=\gamma+\delta$, each $B$-operator creates one particle in the quantum spaces. From this fact and since the state vector (8) is constructed from $N$-layers of the $B$-operators acting on the vacuum state $|\Omega\rangle_{M}$, the state vector (8) is an $N$-particle state for $N \leq M$. To construct a nonvanishing inner product, we introduce the dual $N$-particle state:

$$
\begin{aligned}
\left\langle x_{1} \cdots x_{N}\right| & =\left({ }_{1}\langle 0| \otimes \cdots \otimes{ }_{M}\langle 0|\right) \prod_{j=1}^{N} \sigma_{x_{j}}^{+} \\
& \in \mathscr{F}_{1}^{*} \otimes \cdots \otimes \mathscr{F}_{M}^{*},
\end{aligned}
$$

which are states labelling the configurations of particles $1 \leq$ $x_{1}<x_{2}<\cdots<x_{N} \leq M$.

The projected wavefunctions $W_{M, N}\left(z_{1}, \ldots, z_{N} \mid w_{1}, \ldots\right.$, $\left.w_{M} \mid x_{1}, \ldots, x_{N}\right)$ is defined as the inner product between the state vector (off-shell Bethe vector) $\mid \Phi_{M, N}\left(z_{1}, \ldots, z_{N} \mid\right.$ $\left.\left.w_{1}, \ldots, w_{M}\right)\right\rangle$ and the $N$-particle state $\left\langle x_{1} \cdots x_{N}\right|$ :

$$
\begin{aligned}
& W_{M, N}\left(z_{1}, \ldots, z_{N}\left|w_{1}, \ldots, w_{M}\right| x_{1}, \ldots, x_{N}\right) \\
& \quad=\left\langle x_{1} \ldots x_{N} \mid \Phi_{M, N}\left(z_{1}, \ldots, z_{N} \mid w_{1}, \ldots, w_{M}\right)\right\rangle .
\end{aligned}
$$

See Figure 3 for a pictorial description of (10).

In the next section, we examine the properties of the projected wavefunctions. Here we just remark that the projected wavefunctions of the free-fermion model treated in this paper is not symmetric with respect to the spectral parameters $\left\{z_{1}, \ldots, z_{N}\right\}$. This is in contrast to the case of the projected wavefunctions of the $U_{q}\left(s l_{2}\right)$ six-vertex model, where they are symmetric with respect to the spectral variables, and in that case the Grothendieck polynomials and its quantum group deformation appear. This fact for the properties of the spectral variables of the free-fermion model leads to the Tokuyama formula [10] for the Schur functions, as was first found in [9].

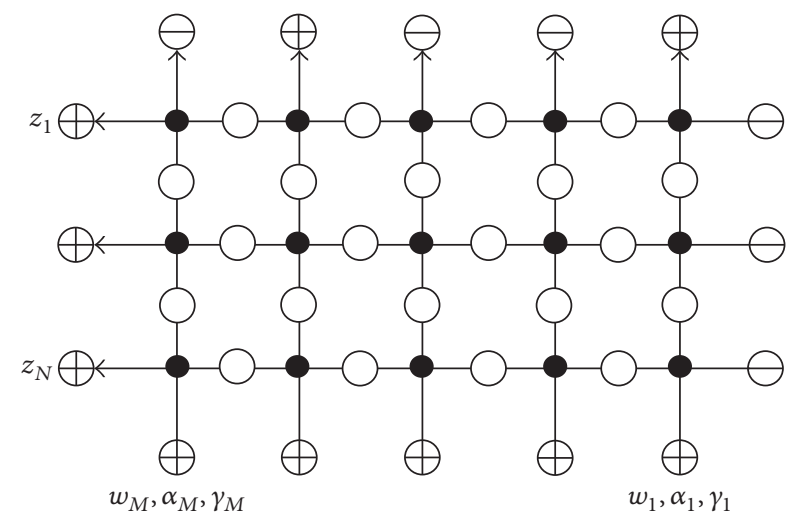

FIGURE 3: The projected wavefunctions $W_{M, N}\left(z_{1}, \ldots, z_{N}\left|w_{1}, \ldots, w_{M}\right|\right.$ $\left.x_{1}, \ldots, x_{N}\right)(10)$. This figure illustrates the cases $M=5, N=3, x_{1}=$ $2, x_{2}=3, x_{3}=5$.

\section{Izergin-Korepin Analysis}

By the Izergin-Korepin analysis, we examine the properties of the projected wavefunctions $W_{M, N}\left(z_{1}, \ldots, z_{N}\left|w_{1}, \ldots, w_{M}\right|\right.$ $\left.x_{1}, \ldots, x_{N}\right)$ in this section.

Proposition 1. The projected wavefunctions $W_{M, N}\left(z_{1}, \ldots\right.$, $\left.z_{N}\left|w_{1}, \ldots, w_{M}\right| x_{1}, \ldots, x_{N}\right)$ satisfy the following properties.

(1) $W_{M, N}\left(z_{1}, \ldots, z_{N}\left|w_{1}, \ldots, w_{M}\right| x_{1}, \ldots, x_{N}\right)$ is a polynomial of degree $N$ in $w_{M}$.

(2) The projected wavefunctions $W_{M, N}\left(z_{\sigma(1)}, \ldots, z_{\sigma(N)}\right.$ $\left.w_{1}, \ldots, w_{M} \mid x_{1}, \ldots, x_{N}\right)$ with the ordering of the spectral parameters permuted $z_{\sigma(1)}, \ldots, z_{\sigma(N)}, \sigma \in S_{N}$ are related to the unpermuted one $W_{M, N}\left(z_{1}, \ldots, z_{N}\left|w_{1}, \ldots, w_{M}\right| x_{1}, \ldots, x_{N}\right)$ by the following relation:

$$
\begin{aligned}
& \prod_{\substack{1 \leq j<k \leq N \\
\sigma(j)>\sigma(k)}}\left(z_{\sigma(j)}+t z_{\sigma(k)}\right) \\
& \cdot W_{M, N}\left(z_{1}, \ldots, z_{N}\left|w_{1}, \ldots, w_{M}\right| x_{1}, \ldots, x_{N}\right)
\end{aligned}
$$




$$
\begin{aligned}
& =\prod_{\substack{1 \leq j<k \leq N \\
\sigma(j)>\sigma(k)}}\left(z_{\sigma(k)}+t z_{\sigma(j)}\right) \\
& \cdot W_{M, N}\left(z_{\sigma(1)}, \ldots, z_{\sigma(N)}\left|w_{1}, \ldots, w_{M}\right| x_{1}, \ldots, x_{N}\right) .
\end{aligned}
$$

(3) The following recursive relations between the projected wavefunctions hold if $x_{N}=M$ :

$$
\begin{aligned}
& \left.W_{M, N}\left(z_{1}, \ldots, z_{N}\left|w_{1}, \ldots, w_{M}\right| x_{1}, \ldots, x_{N}\right)\right|_{w_{M}=\gamma_{M} z_{N}} \\
& \quad=\gamma_{M}^{N} z_{N} \prod_{j=1}^{N-1}\left(z_{j}+t z_{N}\right) \prod_{j=1}^{M-1}\left\{\left(1-\alpha_{j} \gamma_{j}\right) z_{N}+\alpha_{j} w_{j}\right\} \\
& \quad \times W_{M-1, N-1}\left(z_{1}, \ldots, z_{N-1}\left|w_{1}, \ldots, w_{M-1}\right| x_{1}, \ldots, x_{N-1}\right) .
\end{aligned}
$$

When evaluated at $w_{M}=0$, we have

$$
\begin{aligned}
& \left.W_{M, N}\left(z_{1}, \ldots, z_{N}\left|w_{1}, \ldots, w_{M}\right| x_{1}, \ldots, x_{N}\right)\right|_{w_{M}=0} \\
& \quad=0 .
\end{aligned}
$$

If $x_{N} \neq M$, the following factorizations hold for the projected wavefunctions:

$$
\begin{aligned}
& W_{M, N}\left(z_{1}, \ldots, z_{N}\left|w_{1}, \ldots, w_{M}\right| x_{1}, \ldots, x_{N}\right) \\
& =\prod_{j=1}^{N}\left(w_{M}-\gamma_{M} z_{j}\right) \\
& \cdot W_{M-1, N}\left(z_{1}, \ldots, z_{N}\left|w_{1}, \ldots, w_{M-1}\right| x_{1}, \ldots, x_{N}\right) .
\end{aligned}
$$

(4) The following holds for the case $N=1, x_{N}=M$ :

$$
\begin{aligned}
& W_{M, 1}\left(z\left|w_{1}, \ldots, w_{M}\right| M\right) \\
& \quad=w_{M} \prod_{k=1}^{M-1}\left\{\left(1-\alpha_{k} \gamma_{k}\right) z+\alpha_{k} w_{k}\right\} .
\end{aligned}
$$

Proof. Let us first show Properties (1) and (3) for the case $x_{N}=M$.

To show Property (1) when $x_{N}=M$, we first express the projected wavefunctions in terms of the vertical transfer matrix:

$$
\begin{aligned}
& \mathscr{T}_{j}^{N}\left(w_{j} ; z_{1}, \ldots, z_{N}\right) \\
& \quad=L_{a_{1} j}\left(z_{1}, w_{j}, \alpha_{j}, \gamma_{j}\right) \cdots L_{a_{N} j}\left(z_{N}, w_{j}, \alpha_{j}, \gamma_{j}\right) \\
& \quad \in \operatorname{End}\left(W_{a_{1}} \otimes \cdots \otimes W_{a_{N}} \otimes \mathscr{F}_{j}\right) .
\end{aligned}
$$

Using this vertical transfer matrix, the projected wavefunctions can be rewritten as

$$
\begin{aligned}
& W_{M, N}\left(z_{1}, \ldots, z_{N}\left|w_{1}, \ldots, w_{M}\right| x_{1}, \ldots, x_{N-1}, M\right) \\
& =\left\langle\left. 0\right|^{\otimes N}{ }_{M}\langle 1|\left\langle x_{1} \cdots x_{N-1}\right|\right. \\
& \cdot \mathscr{T}_{M}^{N}\left(w_{M} ; z_{1}, \ldots, z_{N}\right) \cdots \mathscr{T}_{1}^{N}\left(w_{1} ; z_{1}, \ldots, z_{N}\right)|1\rangle^{\otimes N} \\
& \cdot|\Omega\rangle_{M}, \\
& \left\langle\left. 0\right|^{\otimes N}={ }_{a_{1}}\langle 0| \otimes \cdots \otimes{ }_{a_{N}}\langle 0|,\right. \\
& |1\rangle^{\otimes N}=|1\rangle_{a_{1}} \otimes \cdots \otimes|1\rangle_{a_{N}} .
\end{aligned}
$$

Inserting the completeness relation in one particle sector

$$
\sum_{j=1}^{N}\left|0^{j-1}, 1,0^{N-j}\right\rangle\left\langle 0^{j-1}, 1,0^{N-j}\right|=\mathrm{Id}
$$

$$
\begin{aligned}
& \left|0^{j-1}, 1,0^{N-j}\right\rangle \\
& =|0\rangle_{a_{1}} \otimes \cdots \otimes|0\rangle_{a_{j-1}} \otimes|1\rangle_{a_{j}} \otimes|0\rangle_{a_{j+1}} \otimes \cdots \\
& \quad \otimes|0\rangle_{a_{N}}, \\
& \left\langle 0^{j-1}, 1,0^{N-j}\right| \\
& ={ }_{a_{1}}\langle 0| \otimes \cdots \otimes{a_{j-1}}\langle 0| \otimes a_{a_{j}}\langle 1| \otimes_{a_{j+1}}\langle 0| \otimes \cdots \\
& \otimes a_{a_{N}}\langle 0|,
\end{aligned}
$$

into (19), we have

$$
\begin{aligned}
& W_{M, N}\left(z_{1}, \ldots, z_{N}\left|w_{1}, \ldots, w_{M}\right| x_{1}, \ldots, x_{N-1}, M\right) \\
& =\sum_{j=1}^{N}\left\langle\left. 0\right|^{\otimes N}{ }_{M}\langle 1| \mathscr{T}_{M}^{N}\left(w_{M} ; z_{1}, \ldots, z_{N}\right)\right. \\
& \cdot\left|0^{j-1}, 1,0^{N-j}\right\rangle|0\rangle_{M} \times\left\langle x_{1} \cdots x_{N-1}\right|\left\langle 0^{j-1}, 1,0^{N-j}\right| \\
& \cdot \mathscr{T}_{M-1}^{N}\left(w_{M-1} ; z_{1}, \ldots, z_{N}\right) \cdots \mathscr{T}_{1}^{N}\left(w_{1} ; z_{1}, \ldots, z_{N}\right) \\
& \cdot|\Omega\rangle_{M-1}|1\rangle^{\otimes N} .
\end{aligned}
$$

In the right-hand side of (21), the parameter $w_{M}$ depends only on $\left\langle\left. 0\right|^{\otimes N}{ }_{M}\left\langle 1\left|\mathscr{T}_{M}^{N}\left(w_{M} ; z_{1}, \ldots, z_{N}\right)\right| 0^{j-1}, 1,0^{N-j}\right\rangle \mid 0\right\rangle_{M}$, whose matrix elements can be easily calculated from its graphical representation as

$$
\begin{gathered}
\left\langle\left. 0\right|^{\otimes N}{ }_{M}\left\langle 1\left|\mathscr{T}_{M}^{N}\left(w_{M} ; z_{1}, \ldots, z_{N}\right)\right| 0^{j-1}, 1,0^{N-j}\right\rangle \mid 0\right\rangle_{M} \\
=w_{M} \prod_{k=1}^{j-1}\left(t w_{M}+\gamma_{M} z_{k}\right) \prod_{k=j+1}^{N}\left(w_{M}-\gamma_{M} z_{k}\right) .
\end{gathered}
$$

Since the matrix elements (22) are a polynomial of degree $N$ in $w_{M}$, one finds that the projected wavefunctions are a polynomial of degree $N$ in $w_{M}$.

Let us next show Property (3) for the case $x_{N}=$ $M$. We first remark that since the projected wavefunctions $W_{M, N}\left(z_{1}, \ldots, z_{N}\left|w_{1}, \ldots, w_{M}\right| x_{1}, \ldots, x_{N}\right)$ are a polynomial of degree $N$ in $w_{M}$, one needs to evaluate $N+1$ distinct points in $w_{M}$ for the Izergin-Korepin trick to be successful. Equation (12) is the result of the evaluation at the point $w_{M}=$ $\gamma_{M} z_{N}$. The $(N-1)$ points $w_{M}=\gamma_{M} z_{j}, j=1, \ldots, N-1$ can be evaluated using Property (2); hence if one shows that certain functions satisfy Property (2), it remains to consider the evaluation at $w_{M}=\gamma_{M} z_{N}$. The evaluation at $w_{M}=\gamma_{M} z_{N}$ essentially gives evaluations at $N$ distinct points. We need one more point to be evaluated. An easy point to be evaluated is $w_{M}=0$, whose result is (13). Let us show these two results of the evaluations.

The recursion relation (12) can be shown as follows. First, from the decomposition (21) and the explicit form of the 
matrix elements (22), one finds that, after the substitution $w_{M}=\gamma_{M} z_{N}$, only the term $j=N$ of the sum in (21) survives and we have

$$
\begin{aligned}
& \left.W_{M, N}\left(z_{1}, \ldots, z_{N}\left|w_{1}, \ldots, w_{M}\right| x_{1}, \ldots, x_{N-1}, M\right)\right|_{w_{M}=\gamma_{M} z_{N}} \\
& =\gamma_{M}^{N} z_{N} \prod_{j=1}^{N-1}\left(z_{j}+t z_{N}\right) \times\left\langle x_{1} \cdots x_{N-1}\right|\left\langle 0^{N-1}, 1\right| \\
& \cdot \mathscr{T}_{M-1}^{N}\left(w_{M-1} ; z_{1}, \ldots, z_{N}\right) \cdots \mathscr{T}_{1}^{N}\left(w_{1} ; z_{1}, \ldots, z_{N}\right) \\
& \cdot|\Omega\rangle_{M-1}|1\rangle^{\otimes N} .
\end{aligned}
$$

Since we can calculate the right-hand side of (23) furthermore as

$$
\begin{aligned}
\left\langle x_{1}\right. & \cdots x_{N-1} \mid\left\langle 0^{N-1}, 1\right| \mathscr{T}_{M-1}^{N}\left(w_{M-1} ; z_{1}, \ldots, z_{N}\right) \\
& \ldots \mathscr{T}_{1}^{N}\left(w_{1} ; z_{1}, \ldots, z_{N}\right)|\Omega\rangle_{M-1}|1\rangle^{\otimes N} \\
& =\left\langle x_{1} \cdots x_{N-1}\right|\left\langle 0^{N-1}\right| \mathscr{T}_{M-1}^{N-1}\left(w_{M-1} ; z_{1}, \ldots, z_{N-1}\right) \\
& \cdots \mathscr{T}_{1}^{N-1}\left(w_{1} ; z_{1}, \ldots, z_{N-1}\right)|\Omega\rangle_{M-1}|1\rangle^{\otimes N-1} \\
& \times a_{a_{N}}\left\langle\left. 1\right|_{M-1}\langle\Omega| L_{a_{N}, M-1}\left(z_{N}, w_{M-1}, \alpha_{M-1}, \gamma_{M-1}\right)\right. \\
& \cdots L_{a_{N}, 1}\left(z_{N}, w_{1}, \alpha_{1}, \gamma_{1}\right)|1\rangle_{a_{N}}|\Omega\rangle_{M-1}
\end{aligned}
$$

$$
\begin{aligned}
& =W_{M-1, N-1}\left(z_{1}, \ldots, z_{N-1}\left|w_{1}, \ldots, w_{M-1}\right| x_{1}, \ldots, x_{N-1}\right) \\
& \cdot \prod_{j=1}^{M-1}\left\{\left(1-\alpha_{j} \gamma_{j}\right) z_{N}+\alpha_{j} w_{j}\right\},
\end{aligned}
$$

we can express the evaluation of $W_{M, N}\left(z_{1}, \ldots, z_{N} \mid w_{1}, \ldots\right.$, $\left.w_{M} \mid x_{1}, \ldots, x_{N}\right)$ at $w_{M}=\gamma_{M} z_{N}$ as

$$
\begin{aligned}
& \left.W_{M, N}\left(z_{1}, \ldots, z_{N}\left|w_{1}, \ldots, w_{M}\right| x_{1}, \ldots, x_{N}\right)\right|_{w_{M}=\gamma_{M} z_{N}} \\
& \quad=\gamma_{M}^{N} z_{N} \prod_{j=1}^{N-1}\left(z_{j}+t z_{N}\right) \prod_{j=1}^{M-1}\left\{\left(1-\alpha_{j} \gamma_{j}\right) z_{N}+\alpha_{j} w_{j}\right\} \\
& \quad \times W_{M-1, N-1}\left(z_{1}, \ldots, z_{N-1}\left|w_{1}, \ldots, w_{M-1}\right| x_{1}, \ldots, x_{N-1}\right) .
\end{aligned}
$$

The evaluation at $w_{M}=0$ (13) can be easily seen by the expansion (21) and the fact that all the matrix elements (22) contain the factor $w_{M}$.

Properties (1) and (3) for the case $x_{N} \neq M$ can be shown much easier. Using the ice rule ${ }_{a}\left\langle\left.\gamma\right|_{j}\langle\delta| L_{a j}(z\right.$, $\left.w_{j}, \alpha_{j}, \gamma_{j}\right)|\alpha\rangle_{a}|\beta\rangle_{j}=0$, one can easily find the following factorization:

$$
\begin{aligned}
& W_{M, N}\left(z_{1}, \ldots, z_{N}\left|w_{1}, \ldots, w_{M}\right| x_{1}, \ldots, x_{N}\right) \\
& \quad=\left\langle\left. 0\right|^{\otimes N}{ }_{M}\left\langle 0\left|\left\langle x_{1} \cdots x_{N}\left|\mathscr{T}_{M}^{N}\left(w_{M} ; z_{1}, \ldots, z_{N}\right) \ldots \mathscr{T}_{1}^{N}\left(w_{1} ; z_{1}, \ldots, z_{N}\right)\right| 1\right\rangle^{\otimes N}\right| \Omega\right\rangle_{M}\right. \\
& \quad=\left\langle\left. 0\right|^{\otimes N}\left\langle 0\left|\mathscr{T}_{M}^{N}\left(w_{M} ; z_{1}, \ldots, z_{N}\right)\right| 0\right\rangle^{\otimes N} \mid 0\right\rangle_{M} \\
& \quad \times\left\langle\left. 0\right|^{\otimes N}\left\langle x_{1} \cdots x_{N}\left|\mathscr{T}_{M-1}^{N}\left(w_{M-1} ; z_{1}, \ldots, z_{N}\right) \ldots \mathscr{T}_{1}^{N}\left(w_{1} ; z_{1}, \ldots, z_{N}\right)\right| 1\right\rangle^{\otimes N} \mid \Omega\right\rangle_{M-1} \\
& =\prod_{j=1}^{N} a_{j}\left\langle\left. 0\right|_{M}\left\langle 0\left|L_{a_{j}, M}\left(z_{j}, w_{M}, \alpha_{M}, \gamma_{M}\right)\right| 0\right\rangle_{a_{j}} \mid 0\right\rangle_{M} \times W_{M-1, N}\left(z_{1}, \ldots, z_{N}\left|w_{1}, \ldots, w_{M-1}\right| x_{1}, \ldots, x_{N}\right) \\
& =\prod_{j=1}^{N}\left(w_{M}-\gamma_{M} z_{j}\right) W_{M-1, N}\left(z_{1}, \ldots, z_{N}\left|w_{1}, \ldots, w_{M-1}\right| x_{1}, \ldots, x_{N}\right) .
\end{aligned}
$$

This shows Properties (1) and (3) for the case $x_{N} \neq M$.

Property (2) can be shown as follows. Using the RLL relation repeatedly, one gets the intertwining relation between the monodromy matrices:

$$
\begin{aligned}
R_{a b}\left(\frac{z_{1}}{z_{2}}\right) T_{a}\left(z_{1} \mid w_{1}, \ldots, w_{M}\right) T_{b}\left(z_{2} \mid w_{1}, \ldots, w_{M}\right) \\
\quad=T_{b}\left(z_{2} \mid w_{1}, \ldots, w_{M}\right) T_{a}\left(z_{1} \mid w_{1}, \ldots, w_{M}\right) \\
\quad \cdot R_{a b}\left(\frac{z_{1}}{z_{2}}\right) .
\end{aligned}
$$

An element of the intertwining relation (27) gives the commutation relation between the $B$-operators:

$$
\left(z_{2}+t z_{1}\right) B\left(z_{1} \mid w_{1}, \ldots, w_{M}\right) B\left(z_{2} \mid w_{1}, \ldots, w_{M}\right)
$$

$$
\begin{aligned}
& =B\left(z_{2} \mid w_{1}, \ldots, w_{M}\right) B\left(z_{1} \mid w_{1}, \ldots, w_{M}\right) \\
& \cdot\left(z_{1}+t z_{2}\right) .
\end{aligned}
$$

Since the projected wavefunctions $W_{M, N}\left(z_{1}, \ldots, z_{N} \mid w_{1}, \ldots\right.$, $\left.w_{M} \mid x_{1}, \ldots, x_{N}\right)(10)$ are constructed from $N$-layers of $B$ operators, the effect of reordering the spectral parameters of the $B$-operators can be traced using the commutation relation (28).

What finally remains is Property (4), which can also easily calculated.

Before presenting the solution in the next section, we explain here why the Izergin-Korepin analysis uniquely defines the projected wavefunctions. The idea is based on the 
following fact: if there are two polynomials $f(w)$ and $g(w)$ of $w$ of degree $N$, and the evaluations of the two polynomials at $N+1$ distinct points are the same $\left(f(w)=g(w)\right.$ for $w=z_{j}$, $j=1, \ldots, N+1$ such that $\left.z_{j} \neq z_{k}, j \neq k\right)$, then the two polynomials are exactly the same, that is, $f(w)=g(w)$ for all $w$. The idea of Izergin-Korepin analysis is to relate the projected wavefunctions $W_{M, N}\left(z_{1}, \ldots, z_{N}\left|w_{1}, \ldots, w_{M}\right| x_{1}, \ldots\right.$, $x_{N}$ ) to smaller ones by using the above fact. The point is to regard $W_{M, N}\left(z_{1}, \ldots, z_{N}\left|w_{1}, \ldots, w_{M}\right| x_{1}, \ldots, x_{N}\right)$ as a polynomial of a single variable $w_{M}$. By Property (1) in Proposition $1, W_{M, N}\left(z_{1}, \ldots, z_{N}\left|w_{1}, \ldots, w_{M}\right| x_{1}, \ldots, x_{N}\right)$ is a polynomial of degree $N$ in $w_{M}$. If $x_{N}=M$, one can evaluate the projected wavefunction at the following $N+1$ points: $w_{M}=\gamma_{M} z_{j}, j=1, \ldots, N, w_{M}=0$. The evaluations at $w_{M}=\gamma_{M} z_{N}$ and $w_{M}=0$ can be obtained by its graphical representation and can be expressed by using the smaller projected wavefunction $W_{M-1, N-1}\left(z_{1}, \ldots, z_{N-1} \mid w_{1}, \ldots\right.$, $\left.w_{M-1} \mid x_{1}, \ldots, x_{N-1}\right)$, which is (12) and (13) of Property (3). The evaluations at $(N-1)$ points $w_{M}=\gamma_{M} z_{j}, j=1, \ldots, N-1$ can be obtained from the evaluation at $w_{M}=\gamma_{M} z_{N}$ using Property (2). This idea is essentially the same with the IzerginKorepin analysis for the domain wall boundary partition functions.

For the case of projected wavefunctions, there is another case we have to consider: the case when $x_{N} \neq M$. For this case, it is easier to connect the projected wavefunctions from its graphical description, and we have (14). Note that the smaller projected wavefunctions connected are $W_{M-1, N}\left(z_{1}, \ldots, z_{N}\left|w_{1}, \ldots, w_{M-1}\right| x_{1}, \ldots, x_{N}\right)$ which are different from the one for the case when $x_{N}=M$.

In both cases $x_{N}=M$ and $x_{N} \neq M$, we are able to connect the projected wavefunctions of different sizes, and, continuing this process successively, the relations can be regarded as recursion relations between projected wavefunctions. For the Izergin-Korepin analysis to be successful such that it gives the uniqueness of the projected wavefunctions, we need the initial condition for the recursion relations, and it is Property (4) in Proposition 1. Hence, if one finds an explicit function satisfying all the properties in Proposition 1, it is the one representing the projected wavefunctions. This is given in the next section.

\section{Generalized Schur Functions}

Definition 2. We define the following symmetric function $F_{M, N}\left(z_{1}, \ldots, z_{N}\left|w_{1}, \ldots, w_{M}\right| x_{1}, \ldots, x_{N}\right)$ which depends on the symmetric variables $z_{1}, \ldots, z_{N}$, complex parameters $w_{1}, \ldots, w_{M}, \alpha_{1}, \ldots, \alpha_{M}, \gamma_{1}, \ldots, \gamma_{M}$ and integers $x_{1}, \ldots, x_{N}$ satisfying $1 \leq x_{1}<\cdots<x_{N} \leq M$ :

$$
\begin{aligned}
F_{M, N} & \left(z_{1}, \ldots, z_{N}\left|w_{1}, \ldots, w_{M}\right| x_{1}, \ldots, x_{N}\right) \\
= & \prod_{j=1}^{N} w_{x_{j}} \frac{1}{\prod_{1 \leq j<k \leq N}\left(z_{k}-z_{j}\right)} \sum_{\sigma \in S_{N}}(-1)^{\sigma} \\
& \cdot \prod_{j=1}^{N} \prod_{k=x_{j}+1}^{M}\left(w_{k}-\gamma_{k} z_{\sigma(j)}\right) \\
& \times \prod_{j=1}^{N} \prod_{k=1}^{x_{j}-1}\left\{\left(1-\alpha_{k} \gamma_{k}\right) z_{\sigma(j)}+\alpha_{k} w_{k}\right\} .
\end{aligned}
$$

The symmetric function $F_{M, N}\left(z_{1}, \ldots, z_{N}\left|w_{1}, \ldots, w_{M}\right|\right.$ $\left.x_{1}, \ldots, x_{N}\right)(29)$ is a generalization of the (factorial) Schur functions. Equation (29) can be rewritten in the form using Young diagrams as

$$
\begin{gathered}
F_{M, N}\left(z_{1}, \ldots, z_{N}\left|w_{1}, \ldots, w_{M}\right| x_{1}, \ldots, x_{N}\right) \\
=\frac{F_{\lambda+\delta}\left(\mathbf{z} \mid w_{1}, \ldots, w_{M}\right)}{\prod_{1 \leq j<k \leq N}\left(z_{j}-z_{k}\right)} .
\end{gathered}
$$

Here, $\mathbf{z}=\left\{z_{1}, \ldots, z_{N}\right\}$ is a set of variables and $\lambda$ denotes a Young diagram $\lambda=\left(\lambda_{1}, \lambda_{2}, \ldots, \lambda_{N}\right)$ with weakly decreasing nonnegative integers $\lambda_{1} \geq \lambda_{2} \geq \cdots \geq \lambda_{N} \geq 0$, and $\delta=(N-1$, $N-2, \ldots, 0) . F_{\mu}(\mathbf{z}|\{\alpha\}|\{\gamma\})$ is an $N \times N$ determinant:

$$
F_{\mu}\left(\mathbf{z} \mid w_{1}, \ldots, w_{M}\right)=\operatorname{det}_{N}\left(f_{\mu_{j}}\left(z_{k} \mid w_{1}, \ldots, w_{M}\right)\right),
$$

where

$$
\begin{aligned}
f_{\mu} & \left(z \mid w_{1}, \ldots, w_{M}\right) \\
& =w_{\mu+1} \prod_{j=1}^{\mu}\left\{\left(1-\alpha_{j} \gamma_{j}\right) z+\alpha_{j} w_{j}\right\} \prod_{j=\mu+2}^{M}\left(w_{j}-\gamma_{j} z\right) .
\end{aligned}
$$

The Young diagrams $\lambda=\left(\lambda_{1}, \lambda_{2}, \ldots, \lambda_{N}\right) \in \mathbb{Z}^{N}(M-N \geq$ $\lambda_{1} \geq \lambda_{2} \geq \cdots \geq \lambda_{N} \geq 0$ ) in form (30) and the sequence of integers $x_{1}, \ldots, x_{N}$ satisfying $1 \leq x_{1}<\cdots<x_{N} \leq M$ in (29) representing $F_{M, N}\left(z_{1}, \ldots, z_{N}\left|w_{1}, \ldots, w_{M}\right| x_{1}, \ldots, x_{N}\right)$ are connected by the translation rule $\lambda_{j}=x_{N-j+1}-N+j-1$, $j=1, \ldots, N$.

In the limit $w_{j}=1, j=1, \ldots, M, \gamma_{j}=0, j=1, \ldots, M$, we can see from form (30) that $F_{M, N}\left(z_{1}, \ldots, z_{N}\left|w_{1}, \ldots, w_{M}\right|\right.$ $\left.x_{1}, \ldots, x_{N}\right)$ reduces to the factorial Schur functions. If one furthermore sets $\alpha_{j}=0, j=1, \ldots, M$, it further reduces to the Schur functions.

We have the following correspondence between the projected wavefunctions of the integrable model and the generalized Schur function $F_{M, N}\left(z_{1}, \ldots, z_{N}\left|w_{1}, \ldots, w_{M}\right|\right.$ $\left.x_{1}, \ldots, x_{N}\right)$.

Theorem 3. The projected wavefunctions of the generalized free-fermion model $W_{M, N}\left(z_{1}, \ldots, z_{N}\left|w_{1}, \ldots, w_{M}\right| x_{1}, \ldots\right.$, $\left.x_{N}\right)$ are explicitly expressed as the product of factors $\prod_{1 \leq j<k \leq N}\left(z_{j}+t z_{k}\right)$ and the symmetric function $F_{M, N}\left(z_{1}, \ldots\right.$, $\left.z_{N}\left|w_{1}, \ldots, w_{M}\right| x_{1}, \ldots, x_{N}\right)$ :

$$
\begin{aligned}
& W_{M, N}\left(z_{1}, \ldots, z_{N}\left|w_{1}, \ldots, w_{M}\right| x_{1}, \ldots, x_{N}\right) \\
& =\prod_{1 \leq j<k \leq N}\left(z_{j}+t z_{k}\right) \\
& \quad \cdot F_{M, N}\left(z_{1}, \ldots, z_{N}\left|w_{1}, \ldots, w_{M}\right| x_{1}, \ldots, x_{N}\right) .
\end{aligned}
$$

In the limits $w_{j}=1, j=1, \ldots, M, \gamma_{j}=0, j=1, \ldots, M$, (33) reduces to the main theorem of Bump-McNamaraNakasuji [22]. Taking the limit $\alpha_{j}=0, j=1, \ldots, M$ furthermore, one gets the main theorem of Bump-BrubakerFriedberg [9]. 
Proof. Let us denote the right-hand side of (33) as $G_{M, N}\left(z_{1}\right.$, $\left.\ldots, z_{N}\left|w_{1}, \ldots, w_{M}\right| x_{1}, \ldots, x_{N}\right)$ :

$$
\begin{aligned}
& G_{M, N}\left(z_{1}, \ldots, z_{N}\left|w_{1}, \ldots, w_{M}\right| x_{1}, \ldots, x_{N}\right) \\
&:=\prod_{1 \leq j<k \leq N}\left(z_{j}+t z_{k}\right) \\
& \cdot F_{M, N}\left(z_{1}, \ldots, z_{N}\left|w_{1}, \ldots, w_{M}\right| x_{1}, \ldots, x_{N}\right) \\
&=\prod_{j=1}^{N} w_{x_{j}} \frac{\prod_{1 \leq j<k \leq N}\left(z_{j}+t z_{k}\right)}{\prod_{1 \leq j<k \leq N}\left(z_{k}-z_{j}\right)} \sum_{\sigma \in S_{N}}(-1)^{\sigma} \\
& \cdot \prod_{j=1}^{N} \prod_{k=x_{j}+1}^{M}\left(w_{k}-\gamma_{k} z_{\sigma(j)}\right) \\
& \quad \times \prod_{j=1}^{N} \prod_{k=1}^{x_{j}-1}\left\{\left(1-\alpha_{k} \gamma_{k}\right) z_{\sigma(j)}+\alpha_{k} w_{k}\right\} .
\end{aligned}
$$

We prove this theorem by showing that $G_{M, N}\left(z_{1}, \ldots, z_{N}\right.$ | $\left.w_{1}, \ldots, w_{M} \mid x_{1}, \ldots, x_{N}\right)$ satisfies all four properties in Proposition 1.

To show Property (1), first note that the factor $\prod_{j=1}^{N} \prod_{k=x_{j}+1}^{M}\left(w_{k}-\gamma_{k} z_{\sigma(j)}\right)$ in $G_{M, N}\left(z_{1}, \ldots, z_{N}\left|w_{1}, \ldots, w_{M}\right|\right.$ $\left.x_{1}, \ldots, x_{N}\right)$ is a polynomial of degree $N-1$ in $w_{M}$ if $x_{N}=M$ and degree $N$ if $x_{N} \neq M$. For the case $x_{N} \neq M$, one sees that the dependence on $w_{N}$ just only comes from this factor. For the case $x_{N}=M$, there is a factor $w_{M}$ coming from the overall factor $\prod_{j=1}^{N} w_{x_{j}}$. Thus, $G_{M, N}\left(z_{1}, \ldots, z_{N}\left|w_{1}, \ldots, w_{M}\right| x_{1}, \ldots, x_{N}\right)$ is a polynomial of degree $N$ in $w_{M}$ for both cases $x_{N}=M$ and $x_{N} \neq M$; hence Property (1) is proved.

Let us next show Property (2). First, note that $F_{M, N}\left(z_{1}\right.$, $\left.\ldots, z_{N}\left|w_{1}, \ldots, w_{M}\right| x_{1}, \ldots, x_{N}\right)$ which is a part of $G_{M, N}\left(z_{1}\right.$, $\left.\ldots, z_{N}\left|w_{1}, \ldots, w_{M}\right| x_{1}, \ldots, x_{N}\right)$ that is symmetric with respect to $z_{1}, \ldots, z_{N}$ since both the denominator $\prod_{1 \leq j<k \leq N}\left(z_{k}-\right.$ $z_{j}$ ) and the numerator

$$
\begin{gathered}
\prod_{j=1}^{N} w_{x_{j}} \sum_{\sigma \in S_{N}}(-1)^{\sigma} \prod_{j=1}^{N} \prod_{k=x_{j}+1}^{M}\left(w_{k}-\gamma_{k} z_{\sigma(j)}\right) \\
\cdot \prod_{j=1}^{N} \prod_{k=1}^{x_{j}-1}\left\{\left(1-\alpha_{k} \gamma_{k}\right) z_{\sigma(j)}+\alpha_{k} w_{k}\right\},
\end{gathered}
$$

are antisymmetric with respect to simple permutations of the spectral parameters $z_{1}, \ldots, z_{N}$. This means

$$
\begin{aligned}
& F_{M, N}\left(z_{1}, \ldots, z_{N}\left|w_{1}, \ldots, w_{M}\right| x_{1}, \ldots, x_{N}\right) \\
& \quad=F_{M, N}\left(z_{\sigma(1)}, \ldots, z_{\sigma(N)}\left|w_{1}, \ldots, w_{M}\right| x_{1}, \ldots, x_{N}\right) .
\end{aligned}
$$

Looking at the other factor $\prod_{1 \leq j<k \leq N}\left(z_{j}+t z_{k}\right)$ which constructs the function $G_{M, N}\left(z_{1}, \ldots, z_{N}\left|w_{1}, \ldots, w_{M}\right| x_{1}, \ldots\right.$, $\left.x_{N}\right)$, we have

$$
\begin{aligned}
& \prod_{\substack{1 \leq j<k \leq N \\
\sigma(j)>\sigma(k)}}\left(z_{\sigma(j)}+t z_{\sigma(k)}\right) \prod_{1 \leq j<k \leq N}\left(z_{j}+t z_{k}\right) \\
& =\prod_{\substack{1 \leq j<k \leq N \\
\sigma(j)>\sigma(k)}}\left(z_{\sigma(k)}+t z_{\sigma(j)}\right) \prod_{1 \leq j<k \leq N}\left(z_{\sigma(j)}+t z_{\sigma(k)}\right) .
\end{aligned}
$$

From (36), (37), and the fact that $G_{M, N}\left(z_{1}, \ldots, z_{N} \mid w_{1}, \ldots\right.$, $\left.w_{M} \mid x_{1}, \ldots, x_{N}\right)$ is defined as a product of $\prod_{1 \leq j<k \leq N}\left(z_{j}+\right.$ $\left.t z_{k}\right)$ and $F_{M, N}\left(z_{1}, \ldots, z_{N}\left|w_{1}, \ldots, w_{M}\right| x_{1}, \ldots, x_{N}\right)$, we have

$$
\begin{aligned}
& \prod_{\substack{1 \leq j<k \leq N \\
\sigma(j)>\sigma(k)}}\left(z_{\sigma(j)}+t z_{\sigma(k)}\right) \\
& \cdot G_{M, N}\left(z_{1}, \ldots, z_{N}\left|w_{1}, \ldots, w_{M}\right| x_{1}, \ldots, x_{N}\right) \\
& =\prod_{\substack{1 \leq j<k \leq N \\
\sigma(j)>\sigma(k)}}\left(z_{\sigma(k)}+t z_{\sigma(j)}\right) \\
& \cdot G_{M, N}\left(z_{\sigma(1)}, \ldots, z_{\sigma(N)}\left|w_{1}, \ldots, w_{M}\right| x_{1}, \ldots, x_{N}\right) .
\end{aligned}
$$

We have shown that $G_{M, N}\left(z_{1}, \ldots, z_{N}\left|w_{1}, \ldots, w_{M}\right| x_{1}\right.$, $\left.\ldots, x_{N}\right)$ satisfies the same relation the projected wavefunctions $W_{M, N}\left(z_{1}, \ldots, z_{N}\left|w_{1}, \ldots, w_{M}\right| x_{1}, \ldots, x_{N}\right)$ must satisfy. Hence Property (2) is proved.

Next we show Property (3). We first prove that the function $G_{M, N}\left(z_{1}, \ldots, z_{N}\left|w_{1}, \ldots, w_{M}\right| x_{1}, \ldots, x_{N}\right)$ satisfies (12) and (13) for the case $x_{N}=M$. To prove (12), we first note that the factor

$$
\prod_{j=1}^{N} \prod_{k=x_{j}+1}^{M}\left(w_{k}-\gamma_{k} z_{\sigma(j)}\right)
$$

in each summand essentially becomes

$$
\prod_{j=1}^{N-1} \prod_{k=x_{j}+1}^{M}\left(w_{k}-\gamma_{k} z_{\sigma(j)}\right) .
$$

Concentrating on the factor $\prod_{j=1}^{N-1}\left(w_{M}-\gamma_{M} z_{\sigma(j)}\right)$ from (40), one finds this factor vanishes unless $\sigma$ satisfies $\sigma(N)=N$ if one substitutes $w_{M}=\gamma_{M} z_{N}$.

Therefore, only the summands satisfying $\sigma(N)=N$ in (29) survive after the substitution $w_{M}=\gamma_{M} z_{N}$. Keeping this in mind, one rewrites $G_{M, N}\left(z_{1}, \ldots, z_{N}\left|w_{1}, \ldots, w_{M}\right|\right.$ $\left.x_{1}, \ldots, x_{N}\right)$ evaluated at $w_{M}=\gamma_{M} z_{N}$ by using the symmetric group $S_{N-1}$ where every $\sigma^{\prime} \in S_{N-1}$ satisfies $\left\{\sigma^{\prime}(1), \ldots, \sigma^{\prime}(N-\right.$ 1) $\}=\{1, \ldots, N-1\}$ as follows:

$$
\begin{aligned}
& \left.G_{M, N}\left(z_{1}, \ldots, z_{N}\left|w_{1}, \ldots, w_{M}\right| x_{1}, \ldots, x_{N}\right)\right|_{w_{M}=\gamma_{M} z_{N}} \\
& \quad=\gamma_{M} z_{N} \prod_{j=1}^{N-1} w_{x_{j}}
\end{aligned}
$$




$$
\begin{aligned}
& \cdot \frac{\prod_{1 \leq j<k \leq N-1}\left(z_{j}+t z_{k}\right) \prod_{j=1}^{N-1}\left(z_{j}+t z_{N}\right)}{\prod_{1 \leq j<k \leq N-1}\left(z_{k}-z_{j}\right) \prod_{j=1}^{N-1}\left(z_{N}-z_{j}\right)} \\
& \times \sum_{\sigma^{\prime} \in S_{N-1}}(-1)^{\sigma^{\prime}} \prod_{j=1}^{N-1} \prod_{k=x_{j}+1}^{M-1}\left(w_{k}-\gamma_{k} z_{\sigma^{\prime}(j)}\right) \prod_{j=1}^{N-1} \gamma_{M}\left(z_{N}\right. \\
& \left.-z_{\sigma^{\prime}(j)}\right) \times \prod_{j=1}^{N-1} \prod_{k=1}^{x_{j}-1}\left\{\left(1-\alpha_{k} \gamma_{k}\right) z_{\sigma^{\prime}(j)}+\alpha_{k} w_{k}\right\} \\
& \cdot \prod_{k=1}^{M-1}\left\{\left(1-\alpha_{k} \gamma_{k}\right) z_{N}+\alpha_{k} w_{k}\right\} .
\end{aligned}
$$

One easily notes that the factors $\prod_{k=1}^{M-1}\left\{\left(1-\alpha_{k} \gamma_{k}\right) z_{N}+\alpha_{k} w_{k}\right\}$ in the sum are independent of the permutation $S_{N-1}^{\prime}$. One also finds that the product of factors $1 / \prod_{j=1}^{N-1}\left(z_{N}-z_{j}\right)$ and $\prod_{j=1}^{N-1} \gamma_{M}\left(z_{N}-z_{\sigma^{\prime}(j)}\right)$ can be simplified as

$$
\begin{aligned}
& \frac{1}{\prod_{j=1}^{N-1}\left(z_{N}-z_{j}\right)} \prod_{j=1}^{N-1} \gamma_{M}\left(z_{N}-z_{\sigma^{\prime}(j)}\right) \\
& =\frac{1}{\prod_{j=1}^{N-1}\left(z_{N}-z_{j}\right)} \prod_{j=1}^{N-1} \gamma_{M}\left(z_{N}-z_{j}\right)=\gamma_{M}^{N-1} .
\end{aligned}
$$

Thus, (41) can be rewritten furthermore as

$$
\begin{aligned}
& \left.G_{M, N}\left(z_{1}, \ldots, z_{N}\left|w_{1}, \ldots, w_{M}\right| x_{1}, \ldots, x_{N}\right)\right|_{w_{M}=\gamma_{M} z_{N}} \\
& =\gamma_{M}^{N} z_{N} \prod_{j=1}^{N-1}\left(z_{j}+t z_{N}\right) \prod_{j=1}^{M-1}\left\{\left(1-\alpha_{j} \gamma_{j}\right) z_{N}+\alpha_{j} w_{j}\right\} \\
& \times \prod_{j=1}^{N-1} w_{x_{j}} \frac{\prod_{1 \leq j<k \leq N-1}\left(z_{j}+t z_{k}\right)}{\prod_{1 \leq j<k \leq N-1}\left(z_{k}-z_{j}\right)} \sum_{\sigma^{\prime} \in S_{N-1}}(-1)^{\sigma^{\prime}} \\
& . \prod_{j=1}^{N-1} \prod_{k=x_{j}+1}^{M-1}\left(w_{k}-\gamma_{k} z_{\sigma^{\prime}(j)}\right) \\
& \quad \times \prod_{j=1}^{N-1} \prod_{k=1}^{x_{j}-1}\left\{\left(1-\alpha_{k} \gamma_{k}\right) z_{\sigma^{\prime}(j)}+\alpha_{k} w_{k}\right\} .
\end{aligned}
$$

Since

$$
\begin{aligned}
& \prod_{j=1}^{N-1} w_{x_{j}} \frac{\prod_{1 \leq j<k \leq N-1}\left(z_{j}+t z_{k}\right)}{\prod_{1 \leq j<k \leq N-1}\left(z_{k}-z_{j}\right)} \sum_{\sigma^{\prime} \in S_{N-1}}(-1)^{\sigma^{\prime}} \\
& \cdot \prod_{j=1}^{N-1} \prod_{k=x_{j}+1}^{M-1}\left(w_{k}-\gamma_{k} z_{\sigma^{\prime}(j)}\right) \\
& \quad \times \prod_{j=1}^{N-1} \prod_{k=1}^{x_{j}-1}\left\{\left(1-\alpha_{k} \gamma_{k}\right) z_{\sigma^{\prime}(j)}+\alpha_{k} w_{k}\right\}
\end{aligned}
$$

$$
\begin{aligned}
& =G_{M-1, N-1}\left(z_{1}, \ldots, z_{N-1}\left|w_{1}, \ldots, w_{M-1}\right| x_{1}, \ldots\right. \\
& \left.x_{N-1}\right)
\end{aligned}
$$

one finds that (43) is nothing but the following recursion relation for the function $G_{M, N}\left(z_{1}, \ldots, z_{N}\left|w_{1}, \ldots, w_{M}\right|\right.$ $\left.x_{1}, \ldots, x_{N}\right)$ :

$$
\begin{aligned}
& \left.G_{M, N}\left(z_{1}, \ldots, z_{N}\left|w_{1}, \ldots, w_{M}\right| x_{1}, \ldots, x_{N}\right)\right|_{w_{M}=\gamma_{M} z_{N}} \\
& =\gamma_{M}^{N} z_{N} \prod_{j=1}^{N-1}\left(z_{j}+t z_{N}\right) \prod_{j=1}^{M-1}\left\{\left(1-\alpha_{j} \gamma_{j}\right) z_{N}+\alpha_{j} w_{j}\right\} \\
& \quad \times G_{M-1, N-1}\left(z_{1}, \ldots, z_{N-1}\left|w_{1}, \ldots, w_{M-1}\right| x_{1}, \ldots, x_{N-1}\right),
\end{aligned}
$$

which is exactly the same recursion relation the projected wavefunctions $W_{M, N}\left(z_{1}, \ldots, z_{N}\left|w_{1}, \ldots, w_{M}\right| x_{1}, \ldots, x_{N}\right)$ must satisfy; hence (12) is shown. Equation (13) can be shown immediately since $G_{M, N}\left(z_{1}, \ldots, z_{N}\left|w_{1}, \ldots, w_{M}\right|\right.$ $\left.x_{1}, \ldots, x_{N}\right)$ evaluated at $w_{M}=0$ becomes zero due to the overall factor $\prod_{j=1}^{N} w_{x_{j}}$ in $F_{M, N}\left(z_{1}, \ldots, z_{N}\left|w_{1}, \ldots, w_{M}\right|\right.$ $\left.x_{1}, \ldots, x_{N}\right)$ and the fact that we are dealing the case $x_{N}=M$.

Now let us examine the case $x_{N} \neq M$. This can be shown in a similar but much simpler way. We rewrite $G_{M, N}\left(z_{1}, \ldots, z_{N}\left|w_{1}, \ldots, w_{M}\right| x_{1}, \ldots, x_{N}\right)$ as

$$
\begin{aligned}
G_{M, N} & \left(z_{1}, \ldots, z_{N}\left|w_{1}, \ldots, w_{M}\right| x_{1}, \ldots, x_{N}\right) \\
= & \prod_{j=1}^{N} w_{x_{j}} \frac{\prod_{1 \leq j<k \leq N}\left(z_{j}+t z_{k}\right)}{\prod_{1 \leq j<k \leq N}\left(z_{k}-z_{j}\right)} \sum_{\sigma \in S_{N}}(-1)^{\sigma} \\
& \cdot \prod_{j=1}^{N} \prod_{k=x_{j}+1}^{M-1}\left(w_{k}-\gamma_{k} z_{\sigma(j)}\right) \prod_{j=1}^{N}\left(w_{M}-\gamma_{M} z_{\sigma(j)}\right) \\
& \times \prod_{j=1}^{N} \prod_{k=1}^{x_{j}-1}\left\{\left(1-\alpha_{k} \gamma_{k}\right) z_{\sigma(j)}+\alpha_{k} w_{k}\right\} .
\end{aligned}
$$

Noting that

$$
\prod_{j=1}^{N}\left(w_{M}-\gamma_{M} z_{\sigma(j)}\right)=\prod_{j=1}^{N}\left(w_{M}-\gamma_{M} z_{j}\right)
$$

we can take this factor out of the sum in (46) and we get

$$
\begin{aligned}
G_{M, N} & \left(z_{1}, \ldots, z_{N}\left|w_{1}, \ldots, w_{M}\right| x_{1}, \ldots, x_{N}\right) \\
= & \prod_{j=1}^{N}\left(w_{M}-\gamma_{M} z_{j}\right) \prod_{j=1}^{N} w_{x_{j}} \frac{\prod_{1 \leq j<k \leq N}\left(z_{j}+t z_{k}\right)}{\prod_{1 \leq j<k \leq N}\left(z_{k}-z_{j}\right)} \\
& \cdot \sum_{\sigma \in S_{N}}(-1)^{\sigma} \prod_{j=1}^{N} \prod_{k=x_{j}+1}^{M-1}\left(w_{k}-\gamma_{k} z_{\sigma(j)}\right)
\end{aligned}
$$




$$
\begin{aligned}
& \times \prod_{j=1}^{N} \prod_{k=1}^{x_{j}-1}\left\{\left(1-\alpha_{k} \gamma_{k}\right) z_{\sigma(j)}+\alpha_{k} w_{k}\right\} \\
& =\prod_{j=1}^{N}\left(w_{M}-\gamma_{M} z_{j}\right) \\
& \cdot G_{M-1, N}\left(z_{1}, \ldots, z_{N}\left|w_{1}, \ldots, w_{M-1}\right| x_{1}, \ldots, x_{N}\right),
\end{aligned}
$$

which is also exactly the recursion relation the projected wavefunctions $G_{M, N}\left(z_{1}, \ldots, z_{N}\left|w_{1}, \ldots, w_{M}\right| x_{1}, \ldots, x_{N}\right)$ must satisfy for the case $x_{N} \neq M$.

Finally it is trivial to check from its definition that

$$
\begin{aligned}
& G_{M, 1}\left(z\left|w_{1}, \ldots, w_{M}\right| M\right) \\
& \quad=w_{M} \prod_{k=1}^{M-1}\left\{\left(1-\alpha_{k} \gamma_{k}\right) z+\alpha_{k} w_{k}\right\}
\end{aligned}
$$

hence Property (4) is shown.

Since we have proved that the function $G_{M, N}\left(z_{1}, \ldots, z_{N}\right.$ | $\left.w_{1}, \ldots, w_{M} \mid x_{1}, \ldots, x_{N}\right)$ satisfies all Properties (1), (2), (3), and (4) in Proposition 1, we conclude that it is the explicit form of the projected wavefunctions $W_{M, N}\left(z_{1}, \ldots, z_{N} \mid w_{1}\right.$, $\left.\ldots, w_{M} \mid x_{1}, \ldots, x_{N}\right)=G_{M, N}\left(z_{1}, \ldots, z_{N}\left|w_{1}, \ldots, w_{M}\right| x_{1}\right.$, $\left.\ldots, x_{N}\right)$.

\section{Conclusion}

In this paper, we studied the generalized free-fermion model in an external field. We applied the Izergin-Korepin analysis on the projected wavefunctions which is a generalization of the Izergin-Korepin analysis on the domain wall boundary partition functions, which was recently done for the case of the $U_{q}\left(s l_{2}\right)$ six-vertex model in [30]. We extracted the properties about the degree, symmetry, recursion relations, and initial conditions the projected wavefunctions satisfy. Next we proved that the product of factors and certain symmetric functions satisfies all the required properties; hence it represents the projected wavefunctions. The result can be regarded as an extension of the Tokuyama formula for the (factorial) Schur functions by Bump-Brubaker-Friedberg [9] and Bump-McNamara-Nakasuji [22].

The result obtained in this paper can also be proved by using the arguments initiated in [9], which views the partition functions as functions of the free parameter in the auxiliary spaces. The Izergin-Korepin analysis used in this paper views the partition functions as functions of inhomogeneous parameters in the quantum spaces. The comparison of the two different ways of arguments seems to be interesting. We use the result obtained in this paper to study algebraic combinatorial properties of the generalized Schur functions in our forthcoming paper [34]. Extending the Izergin-Korepin analysis to other boundary conditions of the generalized free-fermion model is one of the interesting topics regarding this paper. There may be some cases in which the Izergin-Korepin analysis is suitable and some other cases in which the arguments in [9] are useful. We think that developing various techniques are useful for the study of partition functions of integrable lattice models.

\section{Conflicts of Interest}

The author declares that there are no conflicts of interest.

\section{Acknowledgments}

This work was partially supported by Grant-in-Aid for Scientific Research (C) no. 16K05468.

\section{References}

[1] H. Bethe, "Zur Theorie der Metalle," Zeitschrift für Physik, vol. 71, no. 3, pp. 205-226, 1931.

[2] L. D. Faddeev, E. K. Sklyanin, and E. K. Takhtajan, "Quantum inverse problem method. I," Theoretical and Mathematical Physics, vol. 40, p. 194, 1979.

[3] R. J. Baxter, Exactly Solved Models in Statistical Mechanics, Academic Press, London, UK, 1982.

[4] V. E. Korepin, N. M. Bogoliubov, and A. G. Izergin, Quantum Inverse Scattering Method and Correlation Functions, Cambridge Monographs on Mathematical Physics, Cambridge University Press, Cambridge, UK, 1993.

[5] V. Drinfeld, "Hopf algeras and the quantum Yang-Baxter equation," Soviet Mathematics: Doklady, vol. 32, p. 254, 1985.

[6] M. Jimbo, "Aq-difference analogue of U(g) and the Yang-Baxter equation," Letters in Mathematical Physics, vol. 10, no. 1, pp. 6369, 1985.

[7] V. E. Korepin, "Calculation of norms of Bethe wave functions," Communications in Mathematical Physics, vol. 86, no. 3, pp. 391418, 1982.

[8] A. Izergin, "Partition function of the six-vertex model in a finite volume," Soviet Mathematics: Doklady, vol. 32, p. 878, 1987.

[9] B. Brubaker, D. Bump, and S. Friedberg, "Schur polynomials and the Yang-Baxter equation," Communications in Mathematical Physics, vol. 308, no. 2, pp. 281-301, 2011.

[10] T. Tokuyama, "A generating function of strict Gelfand patterns and some formulas on characters of general linear groups," Journal of the Mathematical Society of Japan, vol. 40, no. 4, pp. 671-685, 1988.

[11] S. Okada, "Alternating Sign Matrices and Some Deformations of Weyl's Denominator Formulas," Journal of Algebraic Combinatorics. An International Journal, vol. 2, no. 2, pp. 155-176, 1993.

[12] A. M. Hamel and R. C. King, "Symplectic shifted tableaux and deformations of Weyl's denominator formula for $s p(2 n)$," Journal of Algebraic Combinatorics. An International Journal, vol. 16, no. 3, pp. 269-300 (2003), 2002.

[13] A. M. Hamel and R. C. King, "U-turn alternating sign matrices, symplectic shifted tableaux and their weighted enumeration," Journal of Algebraic Combinatorics. An International Journal, vol. 21, no. 4, pp. 395-421, 2005.

[14] J. H. Perk and C. L. Schultz, "New families of commuting transfer matrices in state vertex models," Physics Letters. A, vol. 84 , no. 8, pp. 407-410, 1981.

[15] B. U. Felderhof, "Direct diagonalization of the transfer matrix of the zero-field free-fermion model," Physica, vol. 65, no. 3, pp. 421-451, 1973. 
[16] S.-Y. Zhao and Y.-Z. Zhang, "Supersymmetric vertex models with domain wall boundary conditions," Journal of Mathematical Physics, vol. 48, no. 2, Article ID 023504, 12 pages, 2007.

[17] O. Foda, A. Caradoc D, M. Wheeler, and M. Zuparic L, "On the trigonometric Felderhof model with domain wall boundary conditions," Journal of Statistical Mechanics, vol. 0703, p. P03010, 2007.

[18] S.-Y. Zhao, W.-L. Yang, and Y.-Z. Zhang, "Determinant representation of correlation functions for the $U_{q}(g 1(11))$ free Fermion model," Journal of Mathematical Physics, vol. 47, no. 1, Article ID 013302, 15 pages, 2006.

[19] D. Ivanov, "Symplectic ice," in Multiple Dirichlet Series, LFunctions and Automorphic Forms, vol. 300 of Progr. Math., pp. 205-222, Birkhäuser Springer, New York, NY, USA, 2012.

[20] B. Brubaker, D. Bump, G. Chinta, and P. E. Gunnells, "Metaplectic whittaker functions and crystals of type B," in Multiple Dirichlet Series, L-Functions and Automorphic Forms, vol. 300 of Progr. Math., pp. 93-118, Birkhäuser Springer, New York, NY, USA, 2012.

[21] S. Tabony J, Deformations of characters, metaplectic Whittaker functions and the Yang-Baxter equation [Ph.D. thesis], Massachusetts Institute of Technology, Cambridge, Mass, USA, 2011.

[22] D. Bump, P. McNamara, and M. Nakasuji, "Factorial schur functions and the Yang-. Baxter equation," Commentarii Mathematici Universitatis Sancti Pauli, vol. 63, p. 23, 2014.

[23] A. M. Hamel and R. C. King, "Tokuyama's identity for factorial Schur $P$ and $Q$ functions," Electronic Journal of Combinatorics, vol. 22, no. 2, Paper 2.42, 30 pages, 2015.

[24] B. Brubaker and A. Schultz, "The six-vertex model and deformations of the Weyl character formula," Journal of Algebraic Combinatorics. An International Journal, vol. 42, no. 4, pp. 917958, 2015.

[25] B. Brubaker, V. Buciumas, and D. Bump, "A Yang-Baxter equation for metaplectic ice," https://arxiv.org/abs/1604.02206.

[26] K. Motegi, "Dual wavefunction of the Felderhof model," Letters in Mathematical Physics, pp. 1-29, 2016.

[27] K. Motegi, “Dual wavefunction of the symplectic ice," https:// arxiv.org/abs/1703.01395.

[28] O. Tsuchiya, "Determinant formula for the six-vertex model with reflecting end," Journal of Mathematical Physics, vol. 39, no. 11, pp. 5946-5951, 1998.

[29] M. Wheeler, "An Izergin-Korepin procedure for calculating scalar products in the six-vertex model," Nuclear Physics. B. Theoretical, Phenomenological, and Experimental High Energy Physics. Quantum Field Theory and Statistical Systems, vol. 852, no. 2, pp. 468-507, 2011.

[30] K. Motegi, "Symmetric functions and wavefunctions of the sixvertex model by Izergin-Korepin analysis," https://arxiv.org/ abs/1703.07924.

[31] K. Motegi and K. Sakai, "Vertex models, TASEP and Grothendieck polynomials," Journal of Physics. A. Mathematical and Theoretical, vol. 46, no. 35, Article ID 355201, 26 pages, 2013.

[32] K. Motegi, "Combinatorial properties of symmetric polynomials from integrable vertex models in finite lattice," https://arxiv .org/abs/1608.02269.

[33] M. Wheeler and P. Zinn-Justin, "Littlewood-Richardson coefficients for Grothendieck polynomials from integrability," in Wheeler M and Zinn-Justin P Littlewood-Richardson coefficients for Grothendieck polynomials from integrability, pp. 1607-02396, arXiv, 1607.02396.
[34] K. Motegi, K. Sakai, and S. Watanabe, in preperation.

[35] M. Wheeler and P. Zinn-Justin, "Refined Cauchy/Littlewood identities and six-vertex model partition functions: III. Deformed bosons," Advances in Mathematics, vol. 299, pp. 543-600, 2016.

[36] D. Betea and M. Wheeler, "Refined Cauchy and Littlewood identities, plane partitions and symmetry classes of alternating sign matrices," Journal of Combinatorial Theory. Series A, vol. 137, pp. 126-165, 2016.

[37] D. Betea, M. Wheeler, and P. Zinn-Justin, "Refined Cauchy/ Littlewood identities and six-vertex model partition functions: II. Proofs and new conjectures," Journal of Algebraic Combinatorics. An International Journal, vol. 42, no. 2, pp. 555-603, 2015.

[38] C. Korff and C. Stroppel, “The sl(n)k-WZNW fusion ring a combinatorial construction and a realisation as quotient of quantum cohomology," Advances in Mathematics, vol. 205, p. $200,2010$.

[39] C. Korff, "Quantum cohomology via vicious and osculating walkers," Letters in Mathematical Physics, vol. 104, no. 7, pp. 771810, 2014.

[40] A. Borodin, "On a family of symmetric rational functions," Advances in Mathematics, vol. 306, pp. 973-1018, 2017.

[41] M. Wheeler and P. Zinn-Justin, "Hall polynomials, inverse Kostka polynomials and puzzles," https://arxiv.org/abs/1603 .01815 . 


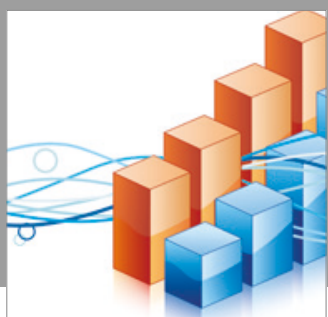

Advances in

Operations Research

vatersals

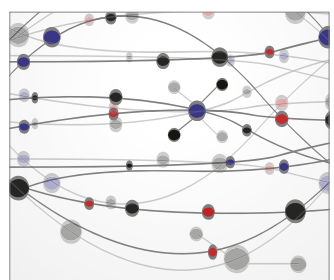

\section{The Scientific} World Journal
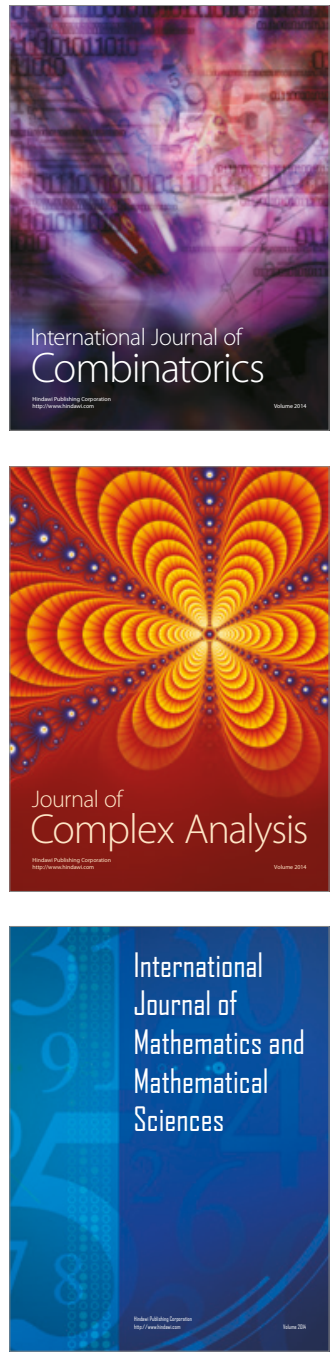
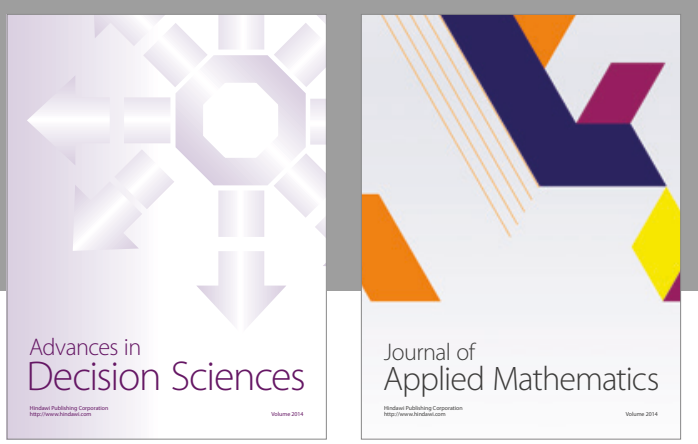

Algebra

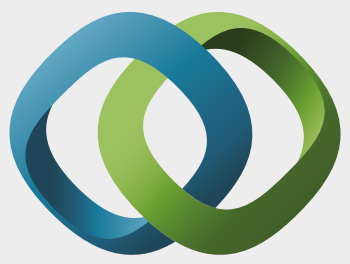

\section{Hindawi}

Submit your manuscripts at

https://www.hindawi.com
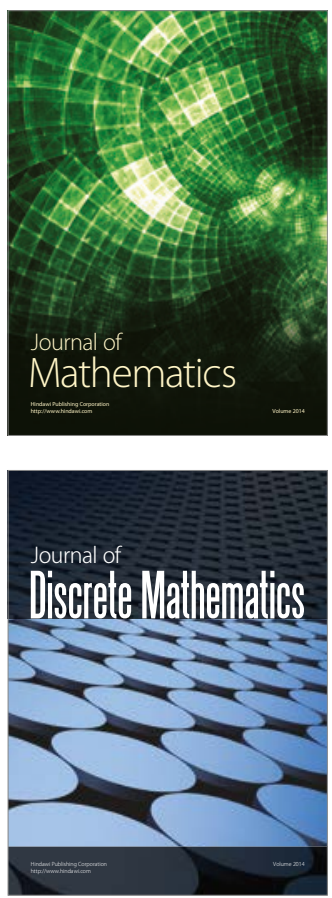

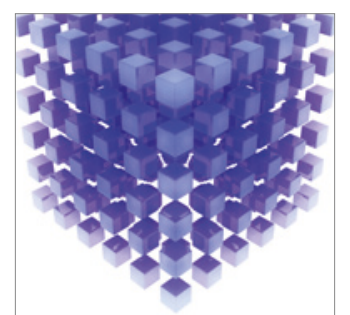

Mathematical Problems in Engineering
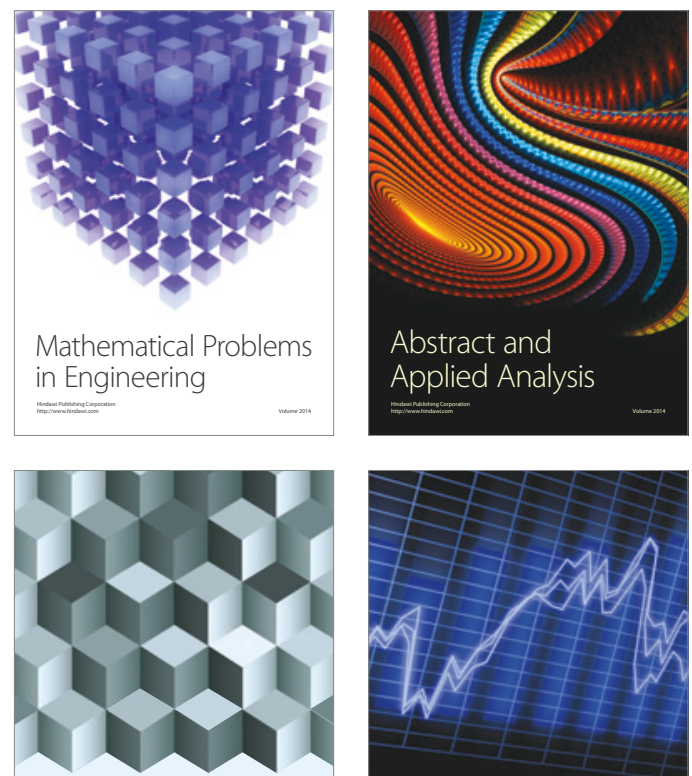

Journal of

Function Spaces

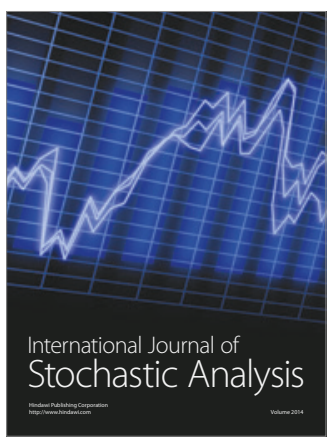

Probability and Statistics
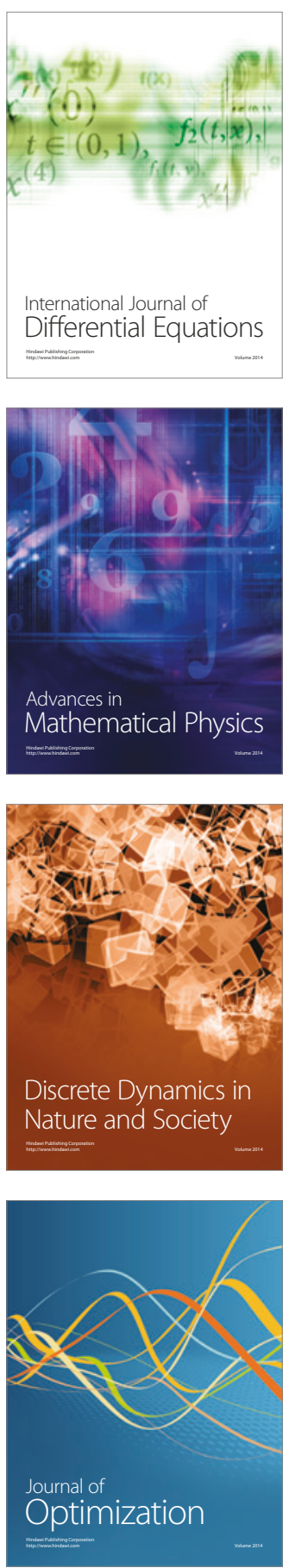